Original research article

\title{
Assessment of cerebral embolism and vascular reserve parameters in patients with carotid artery stenosis
}

\author{
Przemyslaw Puz ${ }^{a, *}$, Anetta Lasek-Bal ${ }^{a}$, Tomasz Urbanek ${ }^{b}$, \\ Zofia Kazibutowska ${ }^{a}$
}

a Department of Neurology, Medical University of Silesia, Professor Leszek Giec Upper Silesian Medical Centre, Katowice, Poland

${ }^{\mathrm{b}}$ Department of General and Vascular Surgery, Medical University of Silesia, Professor Leszek Giec Upper Silesian Medical Centre, Katowice, Poland

\section{A R T I C L E I N F O}

Article history:

Received 31 December 2015

Accepted 23 June 2016

Available online 1 July 2016

Keywords:

Carotid stenosis

Transcranial Doppler

Cerebrovascular reactivity

Vasomotor reactivity test

Microembolic signal

\begin{abstract}
A B S T R A C T
Aim: Carotid artery stenosis can result in the brain tissue injury related to the intracranial aterial flow disturbances as well as microembolic complications. The choice of the proper therapy in patients with carotid artery stenosis, especially asymptomatic, remains still a significant clinical problem. The study aim was an assessment of the cerebral embolism and brain vascular reserve parameters in patients with carotid artery stenosis regarding the occurrence of the clinical symptoms, the degree of stenosis as well as plaque morphology. Methods: The study included 60 patients, with internal carotid artery stenosis. The degree of stenosis, the atherosclerotic plaque surface and morphology were assessed by the means of Duplex Doppler ultrasound. Cerebrovascular reactivity (vasomotor reactivity reserve test and Breath Holding Index) and monitoring of the microembolic signals (MES) were assessed with transcranial Doppler ultrasound examination (TCD).

Results: The vasoreactivity parameters were significantly lower in the group of patients with stenosis $\geq 70 \%$ and in patients with ulcerations on the plaque surface. Microembolic signals were recorded significantly more often in symptomatic patients; in patients with stenosis $\geq 70 \%$; in patients with ulcerations on the plaque surface and those with hypoechogenic plaque structure.

Conclusions: Microembolic signals in patients with symptomatic carotid stenosis are one of the ultrasound features of unstable carotid stenosis. Worse reactivity parameters of the cerebral arteries are associated with the presence of a large degree of carotid artery stenosis.

(C) 2016 Polish Neurological Society. Published by Elsevier Sp. z o.o. All rights reserved.
\end{abstract}

\footnotetext{
* Corresponding author at: Department of Neurology, Clinical Hospital No. 7, Medical University of Silesia, Professor Leszek Giec Upper Silesian Medical Centre, Ziolowa 45, 40-635 Katowice, Poland. Tel.: +48 32 3598306; fax: +48 322029592.

E-mail address: ppuz@tlen.pl (P. Puz).

http://dx.doi.org/10.1016/j.pjnns.2016.06.004

0028-3843/(C) 2016 Polish Neurological Society. Published by Elsevier Sp. z o.o. All rights reserved.
} 


\section{Introduction}

Carotid artery stenosis $>50 \%$ is identified by an ultrasound examination in more than $5 \%$ of people over 65 years of age, twice as often in men [1,2]. According to most investigators, the annual risk of cerebral stroke on the side of stenosis is at the level of $1-5 \%$ [3-5]. The degree of stenosis, the plaque morphology (stability) as well as the probability of thrombus formation at the site of stenosis and also the development of collateral circulation and presence of other risk factors for stroke, all influence on the occurrence of neurological symptoms resulting from internal carotid artery stenosis [6].

The basic method used for diagnostic in patients with carotid artery stenosis is the Duplex Doppler ultrasound examination which allows to assess the degree of stenosis and morphology of stenotic lesions $[7,8]$. The ultrasound characteristics of atherosclerotic plaque associated with higher incidence of cerebral ischemic symptoms include: hypoechogenic, or predominantly hypoechogenic plaque structure and irregularities or ulcerations on the surface of the plaque. However, in the recently published literature, the discussion concerning the definition of the so called "unstable" or ulcerated carotid plaques continues [9-11].

Carotid Duplex Doppler ultrasound is not always a sufficient method to estimate the real risk of cerebral ischemic events. Among available additional diagnostic methods are neurosonological examinations with trancranial Doppler (TCD) ultrasound. TCD enables the assessment of hemodynamic changes in cerebral circulation, development of collateral circulation and detection of microembolic signals (MES) which may be caused by unstable atherosclerotic plaque in carotid arteries [12,13].

Arterio-arterial embolism is the main mechanism of ischemic cerebral stroke in patients with internal carotid stenosis. An important role is also played by hemodynamic changes caused by impaired blood inflow and exhaustion of the compensatory abilities of cerebral circulation associated with the presence of significant internal carotid artery (ICA) stenosis [14,15]. Tests for cerebrovascular reactivity in patients with cerebral circulation insufficiency due to decreased flow resulting from stenosis of the ICA make it possible to assess the vascular reserve. Decrease in the vascular reserve indicates that the adaptation capabilities of the local cerebral flow have been exhausted in response to hypoperfusion and inefficient collateral circulation. Diagnosing such events in patients with ICA stenosis may serve as an additional argument for the necessity of interventional treatment to restore extracranial patency [16-18]. Both factors, microembolisation as well as vascular reserve decrease can coexist in patients with carotid stenosis, however, the prediction of the main factor responsible to the brain ischemic complication in the clinical setting remains still very difficult. The aim of the study was to assess cerebral embolism and vascular reserve parameters with the use of Doppler examination in patients with symptomatic as well as asymptomatic carotid artery stenosis.

\section{Materials and methods}

The study included 60 patients (18 women and 42 men), mean age 66.29 years ( \pm SD 7.77), with symptomatic (38 individuals) or asymptomatic [22] atherosclerotic internal carotid artery stenosis above $50 \%$. In the group of symptomatic patients, neurological symptoms including stroke, reversible ischemic neurological deficit or transient ischemic attack were diagnosed within 6 months before the inclusion into the study (the onset of the neurological complains from 15 to 154 days before the ultrasound examination and carotid artery stenosis diagnosis).

The study inclusion criteria covered: unilateral atherosclerotic internal carotid artery stenosis above $50 \%$ confirmed by the means of with the US Duplex Doppler examination. The exclusion criteria included: non-atherosclerotic stenosis; occlusion or stenosis $\geq 50 \%$ ICA on the contralateral side; potential causes of cerebral embolism other than atherosclerotic lesions in the carotid artery (atrial fibrillation, mitral and/ or aortic valve stenosis, mechanical heart valve, previous myocardial infarction, persistent foramen ovale or another defect, interatrial septal aneurysm, hypo/akinesia of the cardiac walls, cardiac cavity myxoma, thrombus in the left ventricle or atrium, carotid artery dissection or intraluminal thrombosis) - visible in the US examination and brain damage other than of a vascular origin (according to the assessment of the computed tomography or magnetic resonance imaging of the head - imaging study performed in all subjects). All the patients were also rated in relation to the occurrence of stroke risk factors and comorbidities which included: arterial hypertension, ischemic heart disease, diabetes, obesity, peripheral artery disease, chronic kidney disease, lipid disorders and nicotinism.

All the patients included into the study went through: Duplex Doppler ultrasound examination of the carotid and vertebral arteries as well as transcranial Doppler examination of cerebral arteries with assessment of vascular reactivity and monitoring of the middle cerebral artery for the presence of microembolic signals. The duplex carotid and vertebral artery test was performed with the $7.5 \mathrm{MHz}$ linear array probe of PHILIPS ENVISOR C02 along with the assessment of the degree of stenosis and atherosclerotic plaque morphology. Grading of carotid stenosis was based on morphological information (B-mode images, color flow imaging), velocity measurements (in a stenosis and poststenotic segment) and the assessment of collateral flow according to combined criteria for grading internal carotid stenosis published by von Reutern et al. [19].

Plaque echogenicity was based on the Gray-Weale classification and evaluation of the Greyscale Median (GSM) after the normalization of ultrasound images $[20,21]$. Basing on ultrasound examinations the plaque echogenicity was divided into two categories: hypoechogenic (GSM $\leq 25)$ and hyperechogenic (GSM $>25)$. The presence of ulcerations on the surface of atherosclerotic plaque was also assumed as a characteristic of unstable atherosclerotic plaque. Ulceration was determined as a plaque surface niche or crater filled with reversed flow visible in a longitudinal and a transverse plane. Flow disturbances on the plaque surface were imaged using color-coded duplex sonography. Retrogade flow components within a niche were 
recognized due to the inverse (blue) color coding. Ulcerations in tight stenoses (>70\%) were assessed using color-coded duplex examination with adjusting the color scale. Additionally, the plaque surface was delineated using color coding in the power Doppler mode. Flow disturbances and irregularities on the plaque surface visible in both methods were classified as an ulceration. The presence of intraluminar fresh thrombus was excluded basing on the absence of their potential ultrasound features (compressible, mobile, hypoechogenic, "cigar-shaped" intraluminar structure) coexisting with the lack of clinical circumstances enabling the formation of fresh thrombus.

Intracranial arteries were examined with the TCD method by assessing the hemodynamic parameters - mean velocity (Vmean) of flow in the arteries (periorbital arteries, middle cerebral arteries, anterior cerebral arteries, posterior cerebral arteries, vertebral arteries and basilar artery), vasomotor reactivity and by recording microembolic signals. A Pioneer 2002 TC device by EME, equipped with a $2 \mathrm{MHz}$ pulse-wave Doppler ultrasound probe was used in the test. Cerebral embolism was registered for $30 \mathrm{~min}$ in the middle cerebral artery (MCA) at a depth of 55-60 mm on the side of the stenoted artery held in place by headband in 57 patients. The registered embolic signals met the Spencer criteria [22]. In order to evaluate the cerebrovascular reactivity, a test to assess the vasomotor reactivity reserve of the cerebral vessels (VMRr - Vasomotor Reactivity reserve) was used along with Breath Holding Index test (BHI) in accordance with the available standard protocols $[23,24]$. The result of vasomotor reactivity reserve test was reported as the percentage change of mean velocity (Vmean) in the MCA measured during hypocapnia obtained through hyperventilation in relation to hypercapnia caused by breath holding $(\mathrm{VMRr}=[\max$ Vmean $\{$ breath holding $]-$ min Vmean \{hyperventilation\}]/resting Vmean $\times 100$ ). Hyperventilation lasted approximately $2 \mathrm{~min}$; breath holding time was approximately $30 \mathrm{~s}$. The BHI test score was calculated as a percentage increase in mean velocity in the MCA at the time of breath holding in relation to the velocity at rest divided by the time of breath holding according to the Markus-Harrison method [23]. During the reactivity test, the $\mathrm{CO}_{2}$ content in expired air (end - tidal $\mathrm{CO}_{2}$ concentration, $\mathrm{EtCO}_{2}$ ) was monitored continuously using cardiomonitor Philips Intellivue MP30 with capnography measurement module. The prespecified $\mathrm{EtCO}_{2}$ partial pressure cut off values were: $\geq 45 \mathrm{mmHg}$ for hipercapnia (during breath holding) and $\leq 30 \mathrm{mmHg}$ for hypocapnia (during hyperventilation).

The results of Duplex Doppler ultrasound examination were analyzed along with the assessment of carotid stenosis severity, the surface and morphology (echogenicity) of atherosclerotic plaque and the results of transcranial ultrasound tests with the assessment of blood flow velocity in the middle cerebral artery on the side of stenosis, cerebrovascular reactivity indices ( $\mathrm{VMRr}$ and $\mathrm{BHI}$ ) and the presence of microembolic signals. Comparisons were made based on the presence of symptoms, the degree of stenosis, and the plaque echogenicity and surface of atherosclerotic plaque.

All the patients were informed about the aim and course of the study and they have expressed their informed consent. The plan to carry out the study was approved by the Bioethics Committee of the Silesian Medical Academy (decision No: NN6501-90/I/05).

Statistical analysis was performed with the tests set out below. Basic statistical parameters were calculated for interval scale variables. Compliance of the distribution of these variables with normal distribution was verified using the Shapiro-Wilk test. Count and percentage distributions in respect of variable categories were set for nominal variables. Comparisons of mean/median values in the case of interval scale variables were made using the t-test (for variables with normal distribution) or the Mann-Whitney $U$ test (for those variables whose distribution differed from normal distribution). Comparisons of groups in the case of nominal variables were performed using the chi-squared test or Fisher's test, depending on the size of the groups. Interdependencies between the parameters assessing vascular reactivity (VMRr and $\mathrm{BHI}$ ) were presented by calculating the Spearman's rank correlation coefficient and its level of statistical significance. Significance level of $p \leq 0.05$ was assumed to be substantial.

\section{Results}

Stenosis $\geq 70 \%$ and the ulceration on the surface of atherosclerotic plaque occurred significantly more often in the group of patients with symptomatic stenosis than in those with asymptomatic stenosis (Table 1).

Plaque ulceration was found significantly more often in the group of patients with stenosis $\geq 70 \%$ (12 patients, $35.29 \%$ ) than in the group of patients with stenosis of 50-69\% (2 individuals, $7.69 \%, p=0.01$ ) and significantly more often in the patients with hypoechogenic plaques (11 patients, 34.36\%) than in the patients with more stable (hyperechogenic) plaque morphology (3 cases, $10.71 \%, p=0.003$ ).

Microembolic signals in TCD test were recorded significantly more often in the symptomatic stenosis group than in asymptomatic patients; in patients with stenosis $\geq 70 \%$ than in the $50-69 \%$ stenosis group as well as in patients with

Table 1 - Carotid Duplex Doppler ultrasound results in patients with symptomatic and asymptomatic ICA stenosis.

\begin{tabular}{lccc} 
& $\begin{array}{c}\text { Severe stenosis } \\
\text { degree }(\geq 70 \%)\end{array}$ & $\begin{array}{c}\text { Hypoechogenic plaque } \\
\text { in US examination }\end{array}$ & $\begin{array}{c}\text { Ulcerations visible on the plaque } \\
\text { surface in US examination }\end{array}$ \\
\hline Symptomatic $n(\%)$ & $27 / 38(71.1 \%)$ & $23 / 38(60.5 \%)$ & $13 / 38(34.2 \%)$ \\
Asymptomatic $n(\%)$ & $7 / 22(31.8 \%)$ & $9 / 22(40.9 \%)$ & $1 / 22(4.5 \%)$ \\
Level of significance $(p)$ & 0.003 & 0.15 & 0.007 \\
\hline
\end{tabular}


Table 2 - Microembolic signals in different ICA stenosis characteristics.

Presence of microembolic

$p$ signals (MES)

Symptoms of stenosis

Yes $n(\%)$

No $n(\%)$

$18 / 37(48.6 \%)$

0.0006

Degree of stenosis

$60-69 \% n(\%)$

$1 / 20(5 \%)$

$\geq 70 n(\%)$

$4 / 25(16 \%)$

0.01

$15 / 32(46.8 \%)$

Plaque surface in US examination

Without ulceration $n(\%)$

9/43 (20.9\%)

$10 / 14(71.4 \%)$

0.001

With ulceration $n(\%)$

$14 / 31(45.1 \%)$

$5 / 26(19.2 \%)$

Hypoechogenic $n(\%)$

Hyperechogenic $n(\%)$

ulcerations diagnosed on the surface of atherosclerotic plaque and those with hypoechogenic plaques (Table 2).

The mean number of MES was $3.16 \pm 2.01$ (median 3, range 1-8). There were no statistically significant differences in the number of MES regarding the presence of symptoms of the stenosis (mean $3.17 \pm 2.06$, median 2.5 in patients with symptomatic stenosis vs. mean $3 \pm 0$, median 3 in patient with asymptomatic stenosis), the degree of the stenosis, (mean $3.27 \pm 2.02$, median $=3.0$ in patients with stenosis $\geq 70 \%$ vs. mean $2.75 \pm 2.22$, median 2.0 in patients with stenosis $50-69 \%$ ), the plaque surface (mean $3.75 \pm 2.38$, median 3.0 in patients with ulcerated plaques vs. mean $2.73 \pm 1.68$, median 2.0 in patients without ulcerations on the plaque surface) and the plaque echogenicity (mean $3.36 \pm 2.17$, median 3.0 in patients with hypoechogenic plaques vs. mean $2.6 \pm 1.52$, median 2.0 in patients with hyperechogenic plaques).

Parameters evaluating vasoreactivity, $\mathrm{VMRr}$ and $\mathrm{BHI}$, were significantly lower in the group of patients with stenosis $\geq 70 \%$ than in the group with stenosis of $50-69 \%$ and in patients with ulcerations on the surface of atherosclerotic plaque (Table 3). There were no significant differences in MCA mean velocity between the assessed groups (Table 3).
A statistically significant correlation was found between $\mathrm{VMRr}$ and BHI in all patients $(r=0.081, p=0.02)$. The patients who showed microembolic signals had significantly lower values of $\mathrm{VMRr}$ and mean velocity in the MCA than in the group of patients without microembolic signals (Table 4).

\section{Discussion}

Pharmacological and/or interventional treatment applied in patients with carotid artery stenosis is to prevent cerebral ischemic events. The choice of method depends on the result of the patient's clinical assessment and analysis of the morphology of lesion narrowing the artery, also based often on ultrasound examination results [25]. The risk arising from interventional treatment should not be greater than the risk arising from the natural course of the disease and applied pharmacotherapy. In recent years, the effectiveness of conservative treatment has significantly improved in relation to the propagation of the use of statins, antiplatelet drugs and better control over other factors associated with the development of cardiovascular diseases [26].

Diagnostic methods to allow for optimal patient selection criteria are still sought in relation with pharmacological and interventional treatment. In this respect, the importance of transcranial neurosonological examinations along with the assessment of cerebral embolism has been confirmed [12].

The present study identified the following ultrasonographic characteristics of symptomatic atherosclerotic lesions in the carotid arteries: the degree of stenosis $>70 \%$, hypoechogenic structure, ulceration on the surface of the plaque, the presence of microembolic signals recorded in the examination of ipsilateral MCA. The obtained results are consistent with the observations of other authors $[5,27,28]$. Microembolic signals in patients with stenosis of the ICA are considered to be the markers of stenotic atherosclerotic plaque instability. According to Sharma et al. [12], both, the presence of embolic signals and the exhaustion of vascular reserve prove the instability of stenoses also in asymptomatic patients.

Evaluation of cerebrovascular reactivity with the use of TCD test is not included in standard evaluation to qualify patients

\section{Table 3 - Results of vasomotor reactivity tests.}

\begin{tabular}{|c|c|c|c|c|c|c|c|}
\hline Stenosis characteristic & $N$ & $\begin{array}{c}\text { MCA mean }(\mathrm{cm} / \mathrm{s}) \\
\text { Mean } \pm \mathrm{SD}\end{array}$ & $p$ & $\begin{array}{c}\text { VMRr (\%) } \\
\text { Mean } \pm \text { SD }\end{array}$ & $p$ & $\begin{array}{c}\text { BHI } \\
\text { Mean } \pm \text { SD }\end{array}$ & $p$ \\
\hline \multicolumn{8}{|l|}{ Symptoms } \\
\hline Yes & 38 & $44.5 \pm 11.1$ & 0.09 & $54.8 \pm 20.1$ & 0.07 & $0.87 \pm 0.52$ & 0.59 \\
\hline No & 22 & $49.5 \pm 10.7$ & & $62.47 \pm 20.71$ & & $0.96 \pm 0.6$ & \\
\hline \multicolumn{8}{|l|}{ Degree of stenosis } \\
\hline $50-69 \%$ & 26 & $48.7 \pm 12.3$ & 0.14 & $65.5 \pm 20.1$ & 0.001 & $1.0 \pm 0.5$ & 0.05 \\
\hline$\geq 70$ & 34 & $44.5 \pm 9.9$ & & $51.5 \pm 18.9$ & & $0.8 \pm 0.4$ & \\
\hline \multicolumn{8}{|c|}{ Plaque Surface in US examination } \\
\hline With ulcerations & 14 & $44.5 \pm 8.1$ & 0.48 & $47.4 \pm 9.6$ & 0.05 & $0.64 \pm 0.24$ & 0.03 \\
\hline Without ulcerations & 46 & $46.9 \pm 11.2$ & & $60.7 \pm 21.9$ & & $0.98 \pm 0.59$ & \\
\hline \multicolumn{8}{|l|}{ Plaque echogenicity } \\
\hline Hypoechogenic & 32 & $44.7 \pm 10.2$ & 0.24 & $56.2 \pm 17.6$ & 0.89 & $0.88 \pm 0.43$ & 0.51 \\
\hline Hyperechogenic & 28 & $48.1 \pm 12.1$ & & $59.2 \pm 23.6$ & & $0.93 \pm 0.66$ & \\
\hline
\end{tabular}

MCA Vmean, mean velocity in MCA; VMRr, Vasomotor Reactivity reserve test; BHI, Breath Holding Index. 
Table 4 - Vasomotor reactivity tests in patients with and without microembolic signals.

\begin{tabular}{lccccc} 
MES & $\begin{array}{c}\text { VMRr (\%) } \\
\text { Mean } \pm \text { SD }\end{array}$ & $p$ & $\begin{array}{c}\text { BHI } \\
\text { Mean } \pm \text { SD }\end{array}$ & $p$ & $\begin{array}{c}\text { MCA Vmean } \\
\text { Mean } \pm \text { SD }\end{array}$ \\
\hline Present $n=19$ & $45.67 \pm 10.01$ & 0.0008 & $0.74 \pm 0.37$ & 0.13 & $42.37 \pm 10.94$ \\
Absent $n=38$ & $63.78 \pm 21.9$ & $0.98 \pm 0.61$ & & $49.11 \pm 10.83$ \\
\hline \multicolumn{7}{l}{ MCA Vmean, mean velocity in MCA; VMRr, Vasomotor Reactivity reserve test; BHI, Breath Holding Index. } \\
\hline
\end{tabular}

with stenosis of the ICA for surgical treatment, but it can serve as a source of valuable information. In the present study, the patients with stenosis $\geq 70 \%$ and ulcerations on the surface of atherosclerotic plaque showed reduced cerebrovascular reactivity parameters on the side of ICA stenosis in comparison with patients with moderate degree of stenosis and without ulcerations on the plaque surface.

In the available literature, ambiguous reports related to the relationship between cerebrovascular reactivity and the presence of symptoms of ICA can be found [29-31]. In the present study, no significant differences between asymptomatic and symptomatic patients with arterial stenosis were observed in the VMRr analysis. Similar findings were presented by Baracchini et al. [32], who also found that carotid revascularization improves reactivity in patients with unilateral stenosis and occlusion of the ICA on the contralateral side. The authors confirmed that VMRr depends on the degree of stenosis and not on the presence of symptoms.

The main cause of ischemic stroke associated with stenosis of the ICA is the arterio-arterial embolism; in rarer cases (10$15 \%)$ cerebral ischemia results from the reduction in the blood flow volume, if no collateral circulation pathways have been created. Maintenance of proper cerebral flow, despite the presence of ICA stenosis, is possible through mechanisms of cerebral autoregulation. In our study, there were no significant differences in mean flow velocity in MCA between patients with stenosis $\geq 70 \%$ and with stenosis $<70 \%$. Shakur et al. [33] also found no association between MCA flow and the degree of carotid stenosis, using quantitative magnetic resonance angiography as the flow measurement method. Hemodynamic effects of carotid stenosis do not translate directly to distal vasculature. The flow compensation is possible by collateral flow and may not result in significant hemodynamic changes in the blood flow of intracranial arteries. Autoregulation allows to maintain an adequate cerebral flow, but only if it is appropriate and if efficient collateral circulation is sustained $[16,18,29,34,35]$.

Worse cerebrovascular reactivity was observed in the present study in patients with more severe stenosis as well as ulcerations on the surface of the plaque. Worse parameters may be also influenced by the changes associated with the remodeling of arteries in the course of arterial hypertension and atherosclerosis. In patients with microembolic signals (MES) we found lower values of vasomotor reactivity reserve test and lower mean velocity in MCA. The Breath Holding Index (BHI) was also lower, but the difference did not reach statistical significance, probably because of the small number of the studied group and the methodological differences (VMRr test is based on the measurement of mean velocity during hypo- and hypercapnia and in the BHI only hypercapnic phase is included). Lower values of mean flow velocity and reactivity parameters indicate for hypoperfusion, which may impair the clearance (washing-out) of emboli.

Cerebrovascular reactivity assessment as well as plaque feature analysis may have a prospective importance and may be used diagnostic tools in cerebrovascular risk and post stroke disability stratification $[29,36]$. Uzunca et al. found an association of reduced reactivity parameters with disability progression in patients with vascular brain damage. In a sixmonth follow-up, patients with abnormal cerebrovascular reactivity parameters showed worse results on the ADL Barthel scale [37].

Finally, some limitations of the study should be also mentioned including the limited number of patients as well as only unilateral monitoring of the cerebral embolism.

\section{Conclusions}

Microembolic signals in patients with symptomatic carotid stenosis are one of the ultrasound features of unstable carotid stenosis.

Worse reactivity parameters of the cerebral arteries are associated with the presence of a large degree of carotid artery stenosis.

\section{Conflict of interest}

None declared.

\section{Acknowledgement and financial support}

None declared.

\section{Ethics}

The work described in this article has been carried out in accordance with The Code of Ethics of the World Medical Association (Declaration of Helsinki) for experiments involving humans; Uniform Requirements for manuscripts submitted to Biomedical journals.

\section{R E F E R E N C E S}

[1] Sander DDC, Diehm C, Eckstein HH, Kuhnl A, Berkefeld J, Diehl R, et al. Guideline extracranial carotid stenosis, chapter 6: epidemiology. Gefässchirurgie 2012;17(4): 97-501. 
[2] de Weerd M, Greving JP, de Jong AW, Buskens E, Bots ML. Prevalence of asymptomatic carotid artery stenosis according to age and sex: systematic review and metaregression analysis. Stroke 2009;40:1105-13.

[3] Halliday A, Harrison M, Hayter E, Kong X, Mansfield AA, Marro J, et al. 10-year stroke prevention after successful carotid endarterectomy for asymptomatic stenosis (ACST1): a multicentre randomised trial. Lancet 2010;376 (September (9746)):1074-84.

[4] Giannopoulos A, Kakkos S, Abbott A, Naylor AR, Richards T, Mikhailidis DP, et al. Long-term mortality in patients with asymptomatic carotid stenosis: implications for statin therapy. Eur J Vasc Endovasc Surg 2015;50(November (5)):573-82.

[5] Nicolaides AN, Kakkos SK, Griffin M, Sabetai M, Dhanjil S, Tegos T, et al. Severity of asymptomatic carotid stenosis and risk of ipsilateral hemispheric ischaemic events: results from the ACSRS study. Asymptomatic Carotid Stenosis and Risk of Stroke (ACSRS) Study Group. Eur J Vasc Endovasc Surg 2005;30:275-84.

[6] Eckstein HH, Kühnl A, Dörfler A, Kopp IB, Lawall H, Ringleb PA. The diagnosis, treatment and follow-up of extracranial carotid stenosis: a multidisciplinary German-Austrian guideline based on evidence and consensus. Dtsch Arztebl Int 2013;110:468-76.

[7] Brott TG, Halperin JL, Abbara S, Bacharach JM, Barr JD, Bush RL, et al. 2011 ASA/ACCF/AHA/AANN/AANS/ACR/ASNR/ CNS/SAIP/SCAI/S IR/SNIS/SVM/SVS guideline on the management of patients with extracranial carotid and vertebral artery disease. Stroke 2011;42:464-540.

[8] Antignani PL, Benedetti-Valentini F, Aluigi L, Baroncelli TA, Camporese G, Failla G, et al. Diagnosis of vascular diseases. Ultrasound investigations-guidelines. Int Angiol 2012;31 (5 Suppl. 1):1-77.

[9] Naylor AR. Identifying the high-risk carotid plaque. J Cardiovasc Surg (Torino) 2014;55(2 Suppl. 1):11-20.

[10] Huibers A, de Borst GJ, Bulbulia R, Pan H, Halliday A, ACST-1 Collaborative Group. Plaque echolucency and the risk of ischaemic stroke in patients with asymptomatic carotid stenosis within the first asymptomatic carotid surgery trial (ACST-1). Eur J Vasc Endovasc Surg )2015;(December). http:// dx.doi.org/10.1016/j.ejvs.2015.11.013. pii:S1078-5884(15) 00831-X

[11] Salem MK, Bown MJ, Sayers RD, West K, Moore D, Nicolaides A, et al. Identification of patients with a histologically unstable carotid plaque using ultrasonic plaque image analysis. Eur J Vasc Endovasc Surg 2014;4:118-25.

[12] Sharma VK, Tsivgoulis G, Lao AY, Alexandrov AV. Role of transcranial Doppler ultrasonography in evaluation of patients with cerebrovascular disease. Curr Neurol Neurosci Rep 2007;7(January (1)):8-20.

[13] Georgiadis D, Siebler M. Detection of microembolic signals with transcranial Doppler ultrasound. In: Baumgartner RW, editor. Handbook on neurovascular ultrasound. Basel: Karger; 2006. p. 194-205.

[14] Reinhard M, Gerds TA, Grabiak D, Zimmerman PR, Roth M, Guschlbauer B, et al. Cerebral dysautoregulation and the risk of ischemic events in occlusive carotid artery disease. J Neurol 2008;255:1182-9.

[15] Gupta A, Chazen JL, Hartman M, Delgado D, Anumula N, Shao H, et al. Cerebrovascular reserve and stroke risk in patients with carotid stenosis or occlusion: a systematic review and meta-analysis. Stroke 2012;43(November (11)):2884-91.

[16] Bellapart J, Fraser J. Transcranial Doppler assessment of cerebral autoregulation. Ultrasound Med Biol 2009;35:883-93.
[17] Reinhard M, Schwarzer G, Briel M, Altamura C, Palazzo P, King A, et al. Cerebrovascular reactivity predicts stroke in high-grade carotid artery disease. Neurology 2014;83 (October (16)):1424-31.

[18] Vasdekis S, Tsivgoulis G, Athanasiadis D, Andrikopoulou A, Voumvourakis K, Lazaris A, et al. Cerebral reactivity assessment in patients with carotid artery disease: a combined TCD and NIRS Study. J Neuroimaging 2011;20: $1-5$.

[19] von Reutern GM, Goertler MW, Bornstein NM, Del Sette M, Evans DH, Hetzel A, et al. Grading carotid stenosis using ultrasonic methods. Stroke 2012;43(March (3)):916-21.

[20] Gray-Weale AC, Graham JC, Burnett JR, Byrne K, Lusby RJ. Carotid artery atheroma: comparison of preoperative Bmode ultrasound appearance with carotid endarterectomy specimen pathology. J Cardiovasc Surg 1988;29:676-81.

[21] Griffin M, Nicolaides A, Kyriacou E. Normalisation of ultrasonic images of atherosclerotic plaques and reproducibility of grey scale median using dedicated software. Int Angiol 2007;26(December (4)):372-7.

[22] Spencer MP, Thomas GI, Nicholls SC, Sauvage LR. Detection of middle cerebral artery emboli during carotid endarterectomy using transcranial doppler ultrasonography. Stroke 1990;21:415-23.

[23] Markus HS, Harrison MJ. Estimation of cerebrovascular reactivity using transcranial Doppler, including the use of breath-holding as the vasodilatory stimulus. Stroke 1992;23:668-73.

[24] Settakis G, Langyel A, Molnar C, Bereczki D, Csiba L, Fulesdi B. Transcranial Doppler study of the cerebral hemodynamic changes during breath-holding and hyperventilation tests. J Neuroimaging 2002;12:252-8.

[25] Lukasiewicz A, Mindykowski R, Serafin Z. The influence of carotid endarterectomy on cerebral blood flow in significant carotid stenosis-perfusion computed tomography study. Int Angiol 2014;33(August (4)):309-15.

[26] Spence JD, Coates V, Li H, Tamayo A, Muñoz C, Hackam DG, et al. Effects of intensive medical therapy on microemboli and cardiovascular risk in asymptomatic carotid stenosis. Arch Neurol 2010;671:80-6.

[27] Aldemir E, Apaydin M, Varer M, Uluc E. Echolucency of carotid plaques and cerebrovascular events. J Clin Ultrasound 2012;40:399-404.

[28] Rosenkranz M, Wittkugel O, Waiblinger C, Thomalla G, Kruetzelmann A, Havemeister S, et al. Cerebral embolism during carotid artery stenting: role of carotid plaque echolucency. Cerebrovasc Dis 2009;27:443-9.

[29] King A, Serena J, Bornstein NM, Markus HS, ACES Investigators. Does impaired cerebrovascular reactivity predict stroke risk in asymptomatic carotid stenosis? A prospective substudy of the asymptomatic carotid emboli study. Stroke 2011;42(June (6)):1550-5.

[30] Gur A, Bornstein N. Cerebral vasomotor reactivity of bilateral severe carotid stenosis: is stroke unavoidable? Eur J Neurol 2006;13:183-6.

[31] Haussen D, Katsnelsin M, Rodriguez A, Campo N, CampoBustillo I, Romano J, et al. Moderate correlation between breath holding and $\mathrm{CO}_{2}$ inhalation/hyperventilation methods for transcranial Doppler evaluation of cerebral vasoreactivity. J Clin Ulrasound 2012;40:554-8.

[32] Baracchini C, Meneghetti G, Manara R, Ermani M, Ballotta E. Cerebral hemodynamics after contralateral carotid endarterectomy in patients with symptomatic and asymptomatic carotid occlusion: a 10-year follow-up. J Cereb Blood Flow Metab 2006;26:899-905.

[33] Shakur SF, Hrbac T, Alaraj A, Du X, Aletich VA, Charbel FT, et al. Effects of extracranial carotid stenosis on intracranial blood flow. Stroke 2014;45(November (11)):3427-9. 
[34] Reinhard M, Muller T, Guschlbauer B, Timmer J, Hetzel A. Dynamic cerebral autoregulation and collateral flow patterns in patients with severe carotid stenosis or occlusion. Ultrasound Med Biol 2003;29:1105-13.

[35] Soinne L, Helenius J, Tatlisumak T, Saimanen E, Salonen O, Lindsberg $\mathrm{P}$, et al. Cerebral hemodynamics in asymptomatic and symptomatic patients with high-grade carotid stenosis undergoing carotid endarterectomy. Stroke 2003;34:1655-61.
[36] Topakian R, King A, Kwon SU, Schaafsma A, Shipley M, Markus HS, et al. Ultrasonic plaque echolucency and emboli signals predict stroke in asymptomatic carotid stenosis. Neurology 2011;77(August (8)):751-8.

[37] Uzunca I, Asil T, Balci K, Utku U. Evaluation of vasomotor reactivity by transcranial Doppler sonography in patients with acute stroke who have symptomatic intracranial and extracranial stenosis. J Ultrasound Med 2007;26: 179-85. 